\title{
Microvascular Architecture of the Retina in the Japanese Monkey (Macaca fuscata fuscata)
}

\author{
SHIGENORI OKADA AND YoshIKUNI OHTA \\ Department of Anatomy, Osaka Dental University, \\ 5-31 Otemae 1-chome, Chuo-ku, Osaka 540, Japan
}

Received February 4, 1993

\begin{abstract}
The microvascular architecture of the optic region of the retina in the Japanese monkey was revealed under SEM utilizing microcorrosion casts by Ohta's method (1990). At the optic nerve disc, the central retinal artery radiated to four primary retinal arterioles. A dense retinal capillary network between these vessels extended outwards and showed regional differences in density. At the optic nerve disc part, the network developed into a multi-laminar structure containing the radial peripapillary capillaries at the innermost layer. At the macula, the retinal capillaries formed a ring around the central fovea (an avascular area). At the equatorial part, the network was observed to be double-layered: a superficial capillary network (on the vitreous side) located in the nerve fiber layer with a capillary-free zone (CFZ) 30-50 $\mu \mathrm{m}$ in width around arterioles and a deep capillary network (on the choroid side) located at the external nuclear lamina without the CFZ. At the serrata ora, the network diminished to a single layer with coarse ovoid meshes, and the CFZ developed to $50-70 \mu \mathrm{m}$ in width around arterioles. In conclusion, the microvasculature of the retina in the Japanese monkey showed regional variations in proportion to the fine structures of the retinal layers. CFZ occurred since the retinal capillaries were located in the same level as the arterioles.
\end{abstract}

Key Words: microvasculature, retina, microcorrosion casts, Japanese monkey.

\section{INTRODUCTION}

Previously, the fine vasculature of the optic region of the retina and the capillary-free zone (CFZ) were described by His in 1880. Michaelson (1954) demonstrated radial peripapillary capillaries (RPC) by india ink injection. A three-dimensional approach to the capillary network of the retina was taken by Kuwabara and Cogan (1960) utilizing the trypsin-digestion method. Similarly, Shimizu (1970) made microangiographical observations of the retinal capillary architecture. Recently, Ujiie (1976) and Shimizu and Ujiie (1978) examined the retinal microvascular architecture of the monkey employing plastic microcorrosion casts, and discussed the RPC and CFZ. Seki (1987) also studied these vasculatures in the rat using a similar approach. We investigated the microvascular architecture of the retina of the Japanese monkey and elucidated relationship between the double capillary networks, observing from both the vitreous and choroid sides, by means of plastic microcorrosion casts under scanning electron microscope. 


\section{MATERIALS AND METHODS}

Ten adult Japanese monkeys (Macaca fuscata fuscata) supplied by the Cooperative Research Fund of the Primate Institute, Kyoto University, Japan, were used for this study. These animals were anesthetized by an intraperitoneal administration of pentobarbiturate $(40 \mathrm{mg} / \mathrm{kg}$ body weight) and perfused through the ascending aorta with heparinized isotonic sodium chloride solution, 5000 UI/L. For SEM, eight monkeys were injected with acrylic plastic via the common carotid arteries by the plastic injection method (Ohta et al., 1990). After the animals were left for $2 \mathrm{hrs}$ at room temperature for polymerization of the injected plastic, the eyeballs were carefully dissected out. This material was macerated in $5 \% \mathrm{NaOH}$ solution at $60^{\circ} \mathrm{C}$ for $2 \mathrm{hrs}$, washed thoroughly in running water and air-dried. All SEM specimens were sputter-coated with gold in a vacuum evaporator (JFC 1500, JEOL) and observed under a scanning electron microscope (JSM T-300, JEOL) using an accelerating voltage of $5 \mathrm{kv}$ and working distance of $40 \mathrm{~mm}$. The remaining two monkeys were perfused with $2.5 \%$ glutaraldehyde via the common carotid artery, postfixed with $1 \%$ osmic acid, embedded in SPURR's resin and ultrathin-sectioned for transmission electron microscopy (JEM-100S, JEOL) using an accelerating voltage of $80 \mathrm{kv}$.

\section{RESULTS}

To better describe the results, the optic region of the retina of the Japanese monkey was divided into four parts: the optic nerve disc, macula, equator and serrata ora.

The central retinal artery penetrated the center of the scleral part of the lamina cribrosa toward the optical nerve disc, where it divided into four equal arterioles as its primary branches: the superior and inferior nasal retinal arterioles and the superior and inferior temporal arterioles (Fig. 1). Each of these arterioles radiated with the respective satellite venule in the nerve fiber layer. The superior and inferior retinal temporal arterioles gave rise to the superior and inferior macular arterioles, respectively (Fig. 1). These primary arterioles, being small in diameter (80-100 $\mu \mathrm{m})$, ran straight, and venules, being large in diameter (200-300 $\mu \mathrm{m})$, meandered slightly apart from arterioles (Fig. 1). Capillary networks expanding between these arterioles and venules were basically composed of two layers: a superficial (vitreous side) network in the nerve fiber layer (Fig. 1) and a deep (choroid side) network in the internal and external nerve plexiform layers (Fig. 2).

\section{Optic nerve disc (Figs. 3-5)}

Radial peripapillary capillaries (RPC) showed a characteristic appearance in the thick nerve fiber layer of the optic disc (Fig. 1). The RPC of the Japanese monkey, 


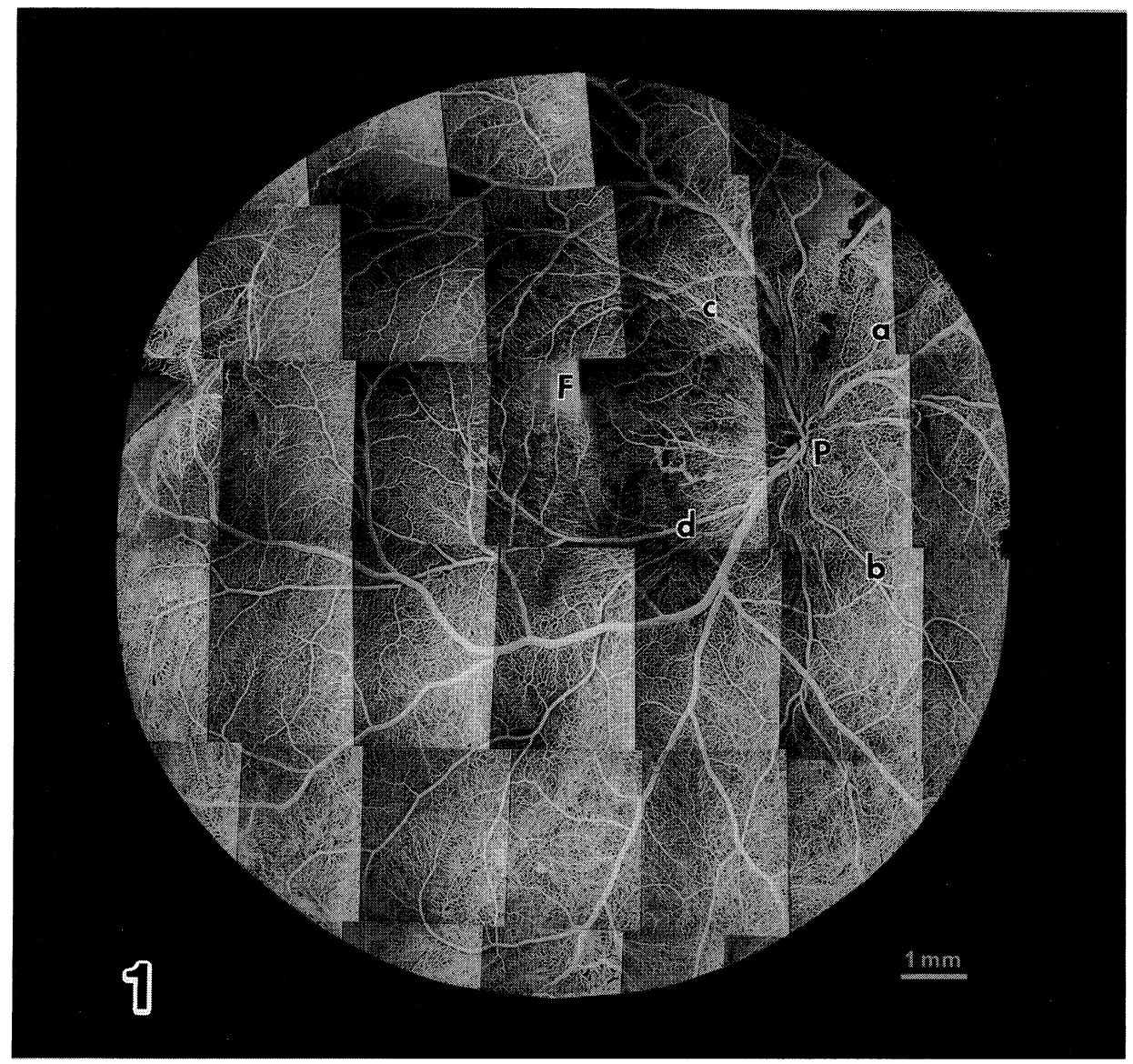

Fig. 1. Anterior (vitreous) view of a corrosion cast of the whole retinal vasculature.

At the optical nerve disc $(\mathrm{P})$, the central retinal artery divides into four primary arterioles; a: Superior nasal retinal, b: Inferior nasal retinal, c: Superior temporal retinal, d: Inferior temporal retinal, F: Central fovea.

appearing as a C-pattern against the macula, as seen in man, diverged from the superficial capillary network and were located superficial to the retinal arterioles and venules (Figs. 3a and 3b). They passed in an arcade for about $200 \mu \mathrm{m}$ in the nerve fiber layer, becoming multilayered close to the optic disc (Fig. 4). The density of the RPC was always in accordance with the density and passage of the nerve fibers between the macula and disc (Figs. 3a and 3b). A capillary network supplying the optic disc communicated with capillaries supplying the optic nerve fasciculus at the optic-nerve head (Figs. 5a and 5b). They became larger in diameter $(15-20 \mu \mathrm{m})$ as soon as they passed straight through the scleral part of the lamina cribrosa and spirally as in the intrinsic vascular system of other peripheral nerve fasciculi (Figs. 5a and 5b). The arterial circle (of Zinn) was not observed, but 


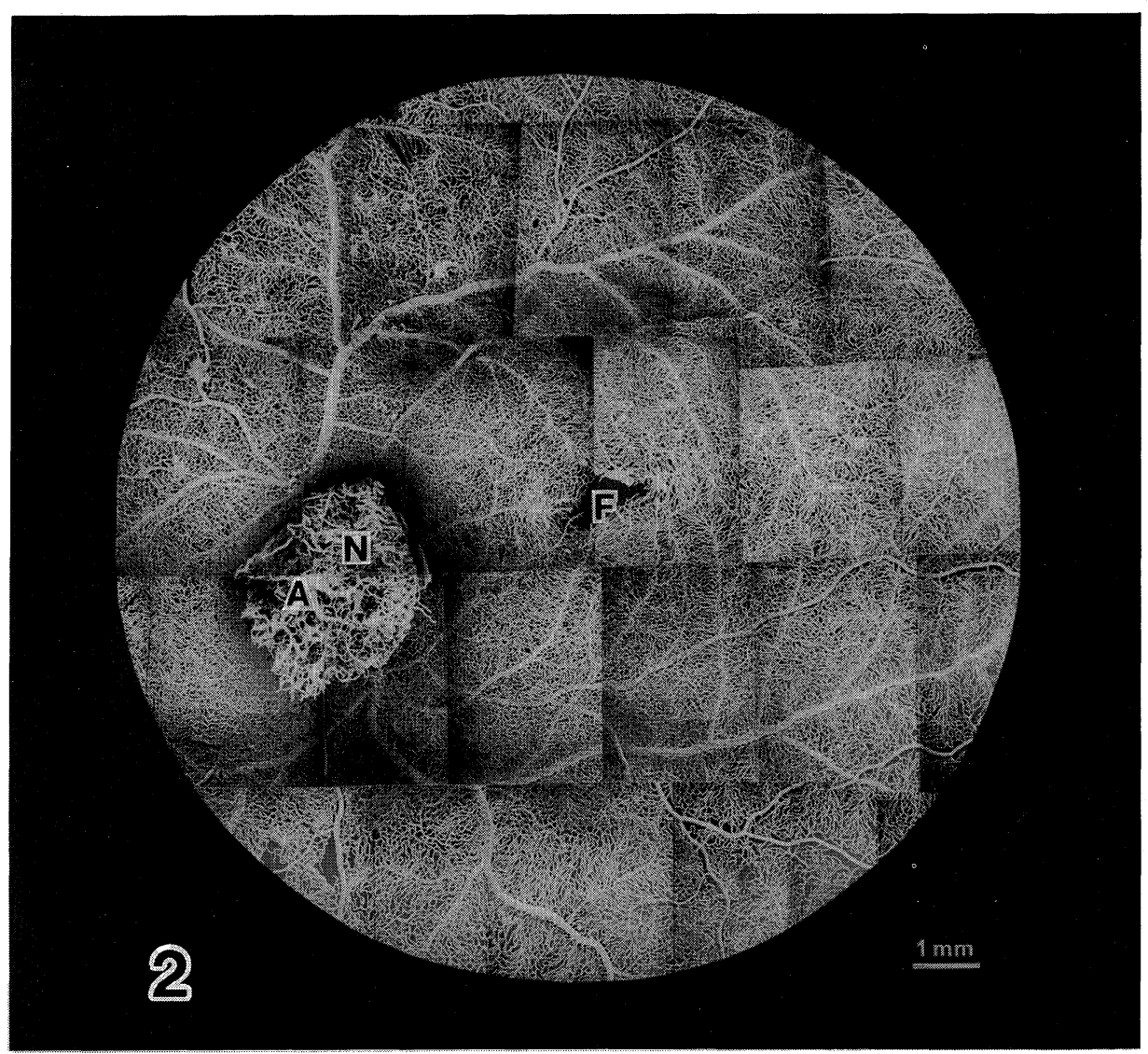

Fig. 2. Posterior (choroid) view of the same specimen in Fig. 1. Fine capillary network expands on the choroid side of the retina. F: Central fovea, N: Optic-nerve head, A: Central retinal artery.

fine branches were distributed to the optic nerve fasciculus.

\section{Macula (Figs. 3a and 6)}

In this region, the vascular architecture faded toward the central fovea since the nerve fiber layer disappeared up to the internal nerve plexiform layer in the central fovea.

Around the macula, the superior and inferior macular arterioles gave rise to capillaries, meshworks of which concentrically surrounded the fovea (Fig. 3a). Also, the superficial capillary layer (on the vitreous side) gradually disappeared close to the fovea. Accordingly, only the deep capillary layer (on the choroid side) surrounded the fovea and formed a capillary ring surrounding an avascular area (Fig. 6a). Blood from the ring drained into four radiating venules (Fig. 6b). 

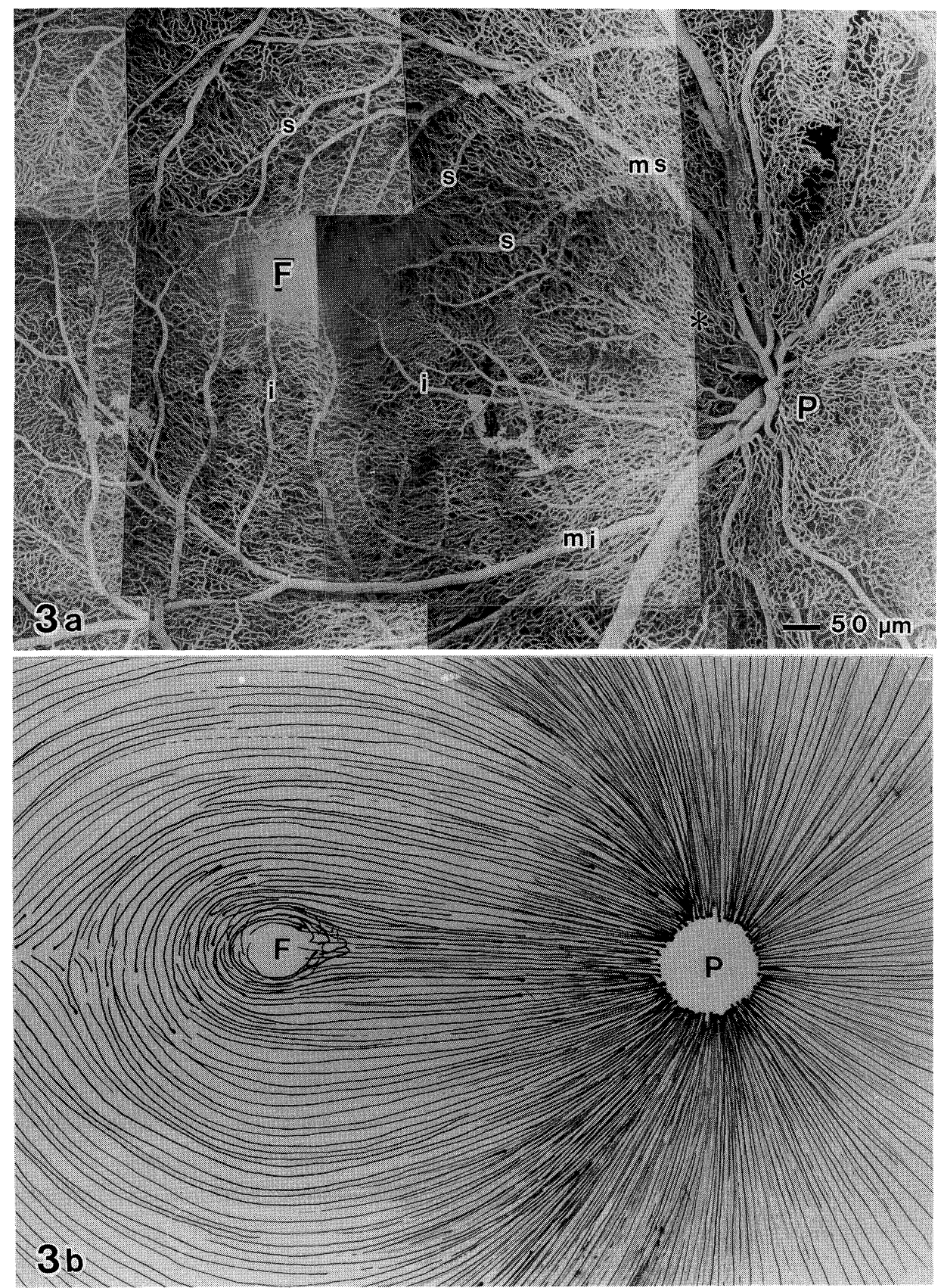

Fig. 3a. A close-up of the optical nerve disc (P) and macula areas.

Fig. 3b. Drawing of the nerve fibers between these areas.

The superior (ms) and inferior (mi) macular arterioles diverged from the superior (s) and inferior (i) temporal retinal arterioles, respectively. The RPC (*) figures $\mathrm{C}$ facing to the macula like a pattern of the nerve fiber. The central fovea $(\mathrm{F})$ is observed as an avascular area. 


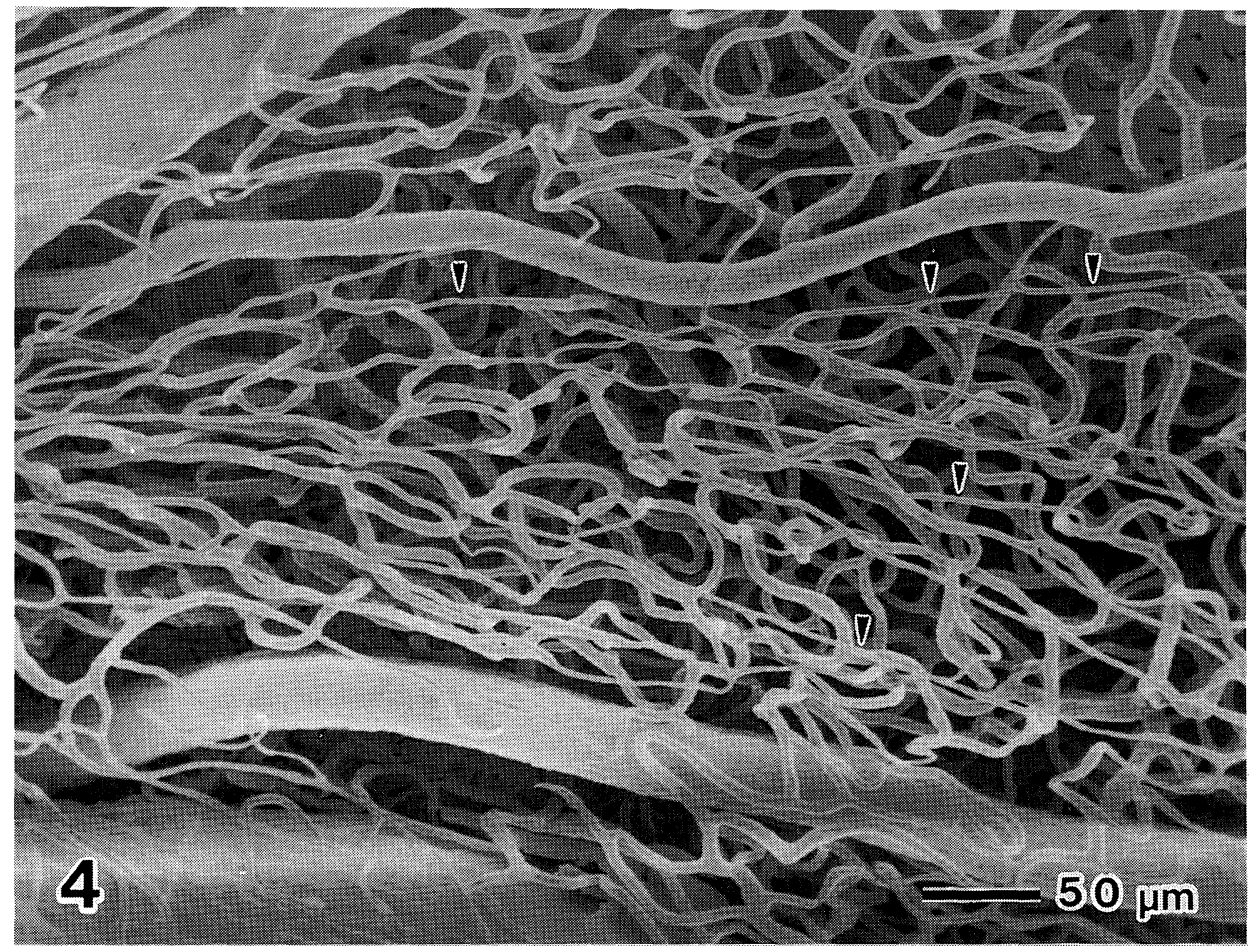

Fig. 4. A close-up of the optical nerve disc area.

The RPC (arrowheads) arches for about $200 \mu \mathrm{m}$ distant from and along the passage of the nerve fibers.

\section{Equator (Figs. 7 and 8)}

Typically double-layered capillary networks extended between the macula and equatorial parts (Figs. 7a and 7b). Capillaries diverging from each arteriole in regular intervals and at right angles to the parent vessel showed repeated branching and anastomosing with adjacent capillaries, forming the superficial capillary network (Fig. 7a). These capillaries were usually large in diameter $(15-20 \mu \mathrm{m})$ and formed large meshworks (about $200 \mu \mathrm{m}$ ) in the nerve fiber layer (Fig. 7a). The deep capillary network was constructed of fine capillaries with a slight tortuosity and did not extend in a plane but undulated in small meshworks (about $150 \mu \mathrm{m}$ ) in the internal and external nerve plexiform layers (Fig. 7b). The two capillary networks communicated with each other. Blood from the deep network drained into the venules in the nerve fiber layer. A capillary-free zone (CFZ), of 30-50 $\mu \mathrm{m}$ in width was observed around arteriolar branches, but no CFZ was seen around venules (Fig. 7a).

Endothelial cells of these networks were surrounded by a thick, continuous basement membrane with a flat nucleus and pericytes. Capillaries in the optic region of the retina appeared as a continuous endothelial wall (Fig. 8). 

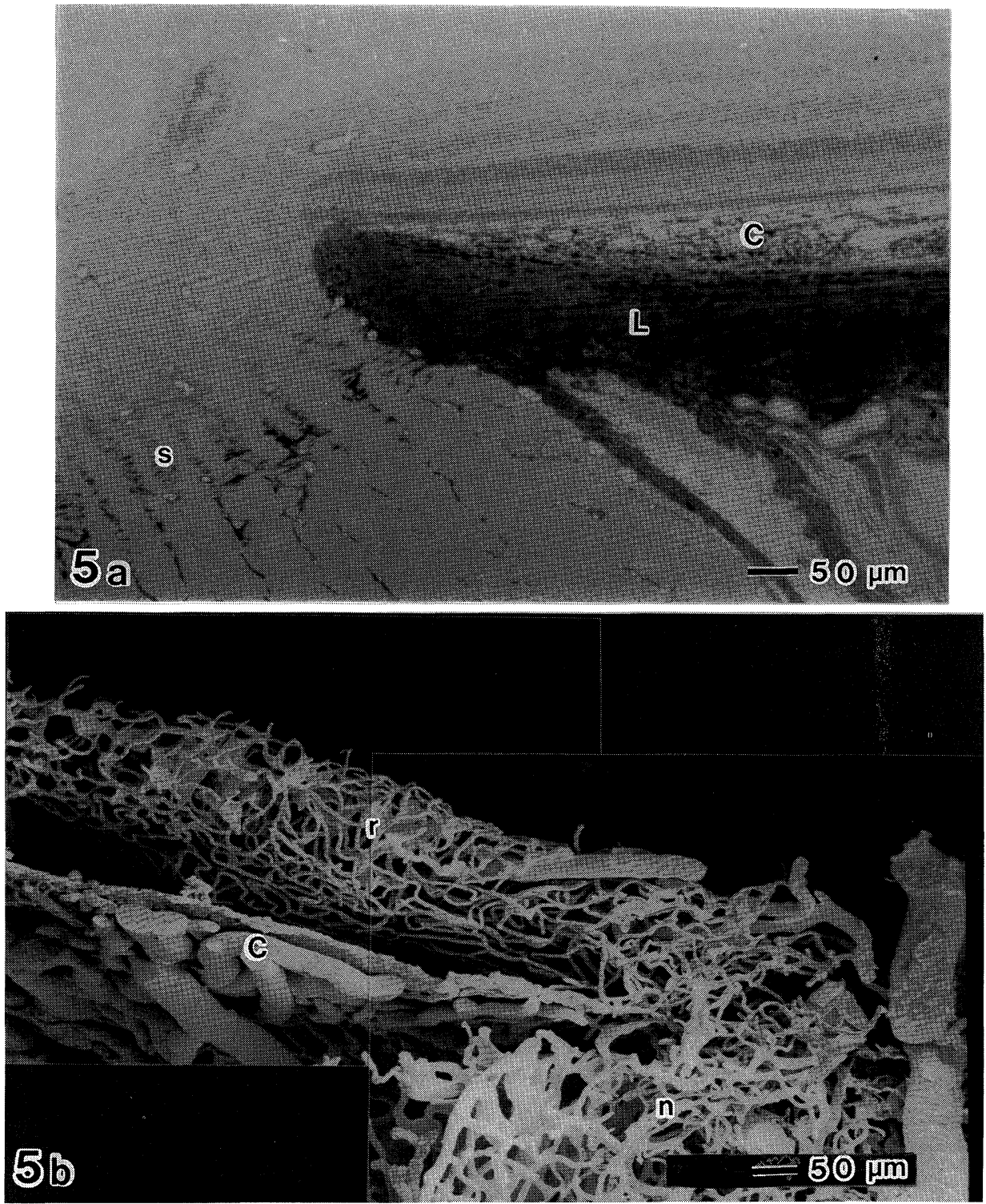

Fig. 5a. Light microscopic photography of the optic-nerve head.

Fig. 5b. A sagittal section of a corrosion cast through the optic-nerve head.

The retinal capillary network (r) supplying the optic disc communicates with capillaries (n) supplying the optic nerve fasciculus at the optic-nerve head via the scleral part (s) of the lamina cribrosa (L). C: Choroid layer.

\section{Serrata ora (Figs. 9 and 10)}

Close to the serrata ora, the internal and external nerve plexiform layers adhered to each other, and the boundary between the nerve cell and fiber layers gradually 

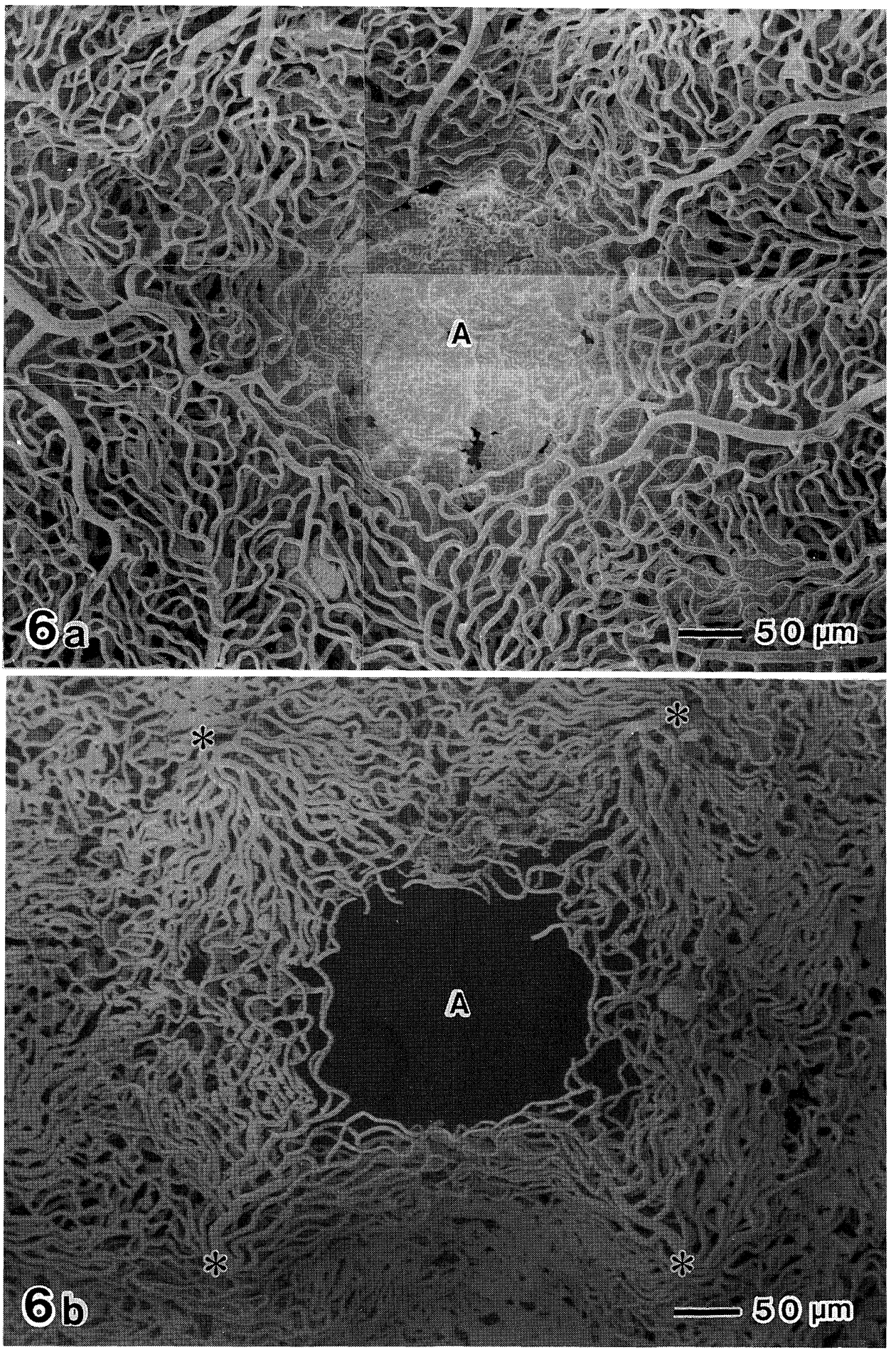
Figs. 6a and 6b. An avascular area in the macula area viewed from vitreous (6a) and choroid (6b) sides.

The superficial capillary around the macula becomes fading toward the central fovea. Only the deep capillary network forms a capillary ring surrounding the avascular area (A). Blood from the ring drains into four radiating venules $\left(^{*}\right)$.

disappeared. According to these changes, the double-layered capillary networks adhered to form a single network (Figs. 9a and 9b). Capillaries of this network diverged from the arterial branches at 700-900 $\mu \mathrm{m}$ intervals, and branched repeatedly to form a capillary network (Fig. 9a). Its meshworks were coarse (200-300 $\mu \mathrm{m})$, spreading in a plane with a wide CFZ (50-70 $\mu \mathrm{m}$ in width) around arterial vessels, but no CFZ was present around venous vessels (Figs. 9a and 9b). Blood from the capillaries drained into venules passing on the choroid side (Fig. 9b). Meshworks around the serrata ora were longitudinally more coarse $(300-500 \mu \mathrm{m})$ and elongated in the meridian direction, with capillary loops along the serrata ora (Fig. 10).

\section{DISCUSSION}

Microvascular architecture of the retina has been examined by means of many research devices, such as the india ink injection, the tripsin digestion, the fluorecent microradiography, microangiography and serial histological reconstruction, etc. It, however, has been difficult to make three dimensional observations on both the vitreous and the choroid sides or separating from each other in one specimen. Microcorrosion technique for elucidating microvasculature three-dimensionally is most helpful to examine the retinal vascular architecture by both the vitreous (internal) and choroid (external) sides of the retina, so that the double layered structure of the retinal vasculature was demonstrated first in this paper.

\section{Optic nerve disc}

Since the existence of the radial peripapillary capillaries (RPC) was indicated by Michaelson (1954), differences on RPC between animal species have been investigated. Henkind (1967) made a comprehensive survey of previous studies on the RPC and designated its differential diagnosis. However, the RPC may be not a spherical vasculature, as reported by Ujiie (1976) and Shimizu and Ujiie (1978), but a kind of satellite blood vessels of the nerve fiber. Nerve fibers of the optic nerve disc part become thicker in close to the optic disc, so that capillary networks in it become multilayered proportionally. Morphological variations between animal species are determined by passages and density of the nerve fibers. Besides the passage is remarkablly influenced by the presence and form of the optic nerve disc.

On the vasculature of the optic-nerve head, there have been many discrepancy in 

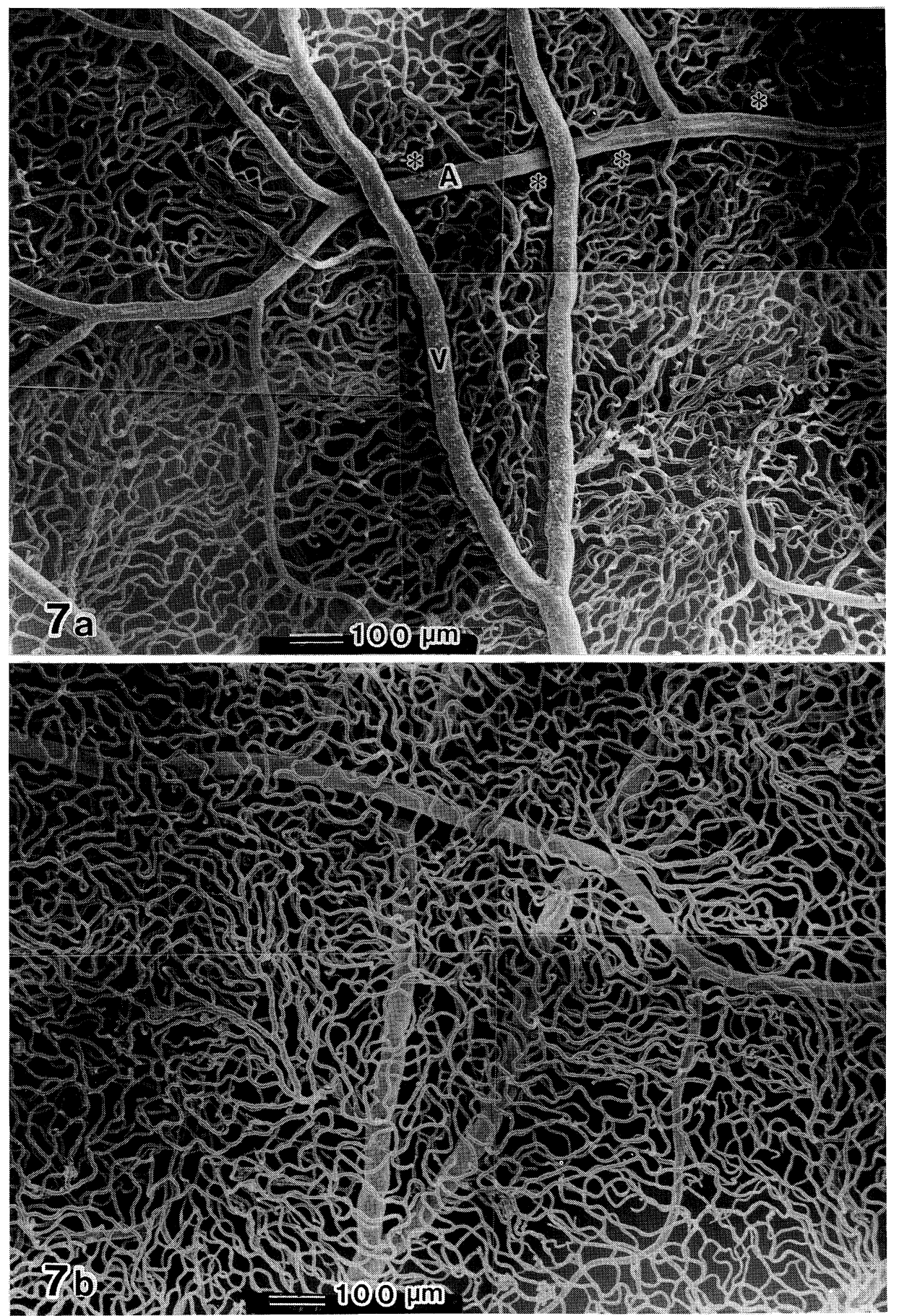
Fig. 7a and 7b. Double-layered capillary networks viewed from vitreous (7a) and choroid (7b) sides. Superficial capillary forms a large meshwork in the nerve fiber layer. A capillary-free zone $(*)$ is observed around arteriole (A), but no CFZ around venule (V). Deep capillary network is undulated in the internal and external nerve plexiform layers.

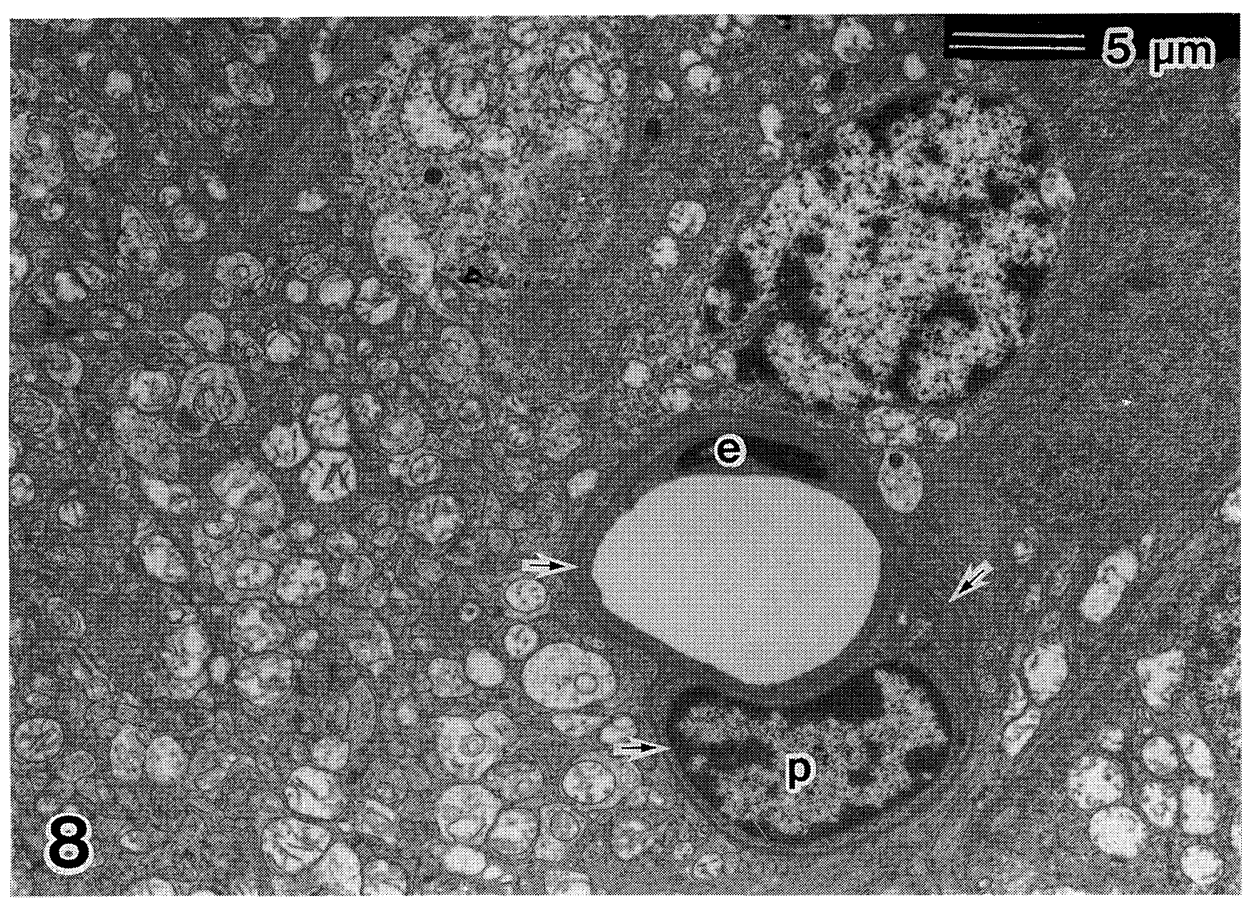

Fig. 8. TEM photography of a retinal capillary.

Retinal capillary appears as a continuous type with a flat nucleus (e) and a thick basement membrane (arrows). P: Pericyte.

investigators' opinions on correlations between the sclera vasculature and circulation in the optic nerve and between the vitreous vasculature around the disc and the capillary network of the optic-nerve head. As described by Wybar (1956), Ernest and Potts (1968), Anderson (1970), Weiter and Ernest (1974), Anderson and Braverman(1976), and Lieberman et al. (1976), an arterial circle of Zinn is neither observed in the Japanese monkey, nor ramifications to the optic nerve from the sclera vasculature as shown by Araki (1976). Correlations between the capillary networks around the disc and the network of the optic-nerve head were observed as a communication with each other by Lieberman et al. (1976) and Ojima (1977), although this was denied by Ernest and Potts (1968) and Araki (1976). In this paper, an apparent demarcation was recognized between the capillary network around the disc and the capillary of the optic nerve in the Japanese monkey. 


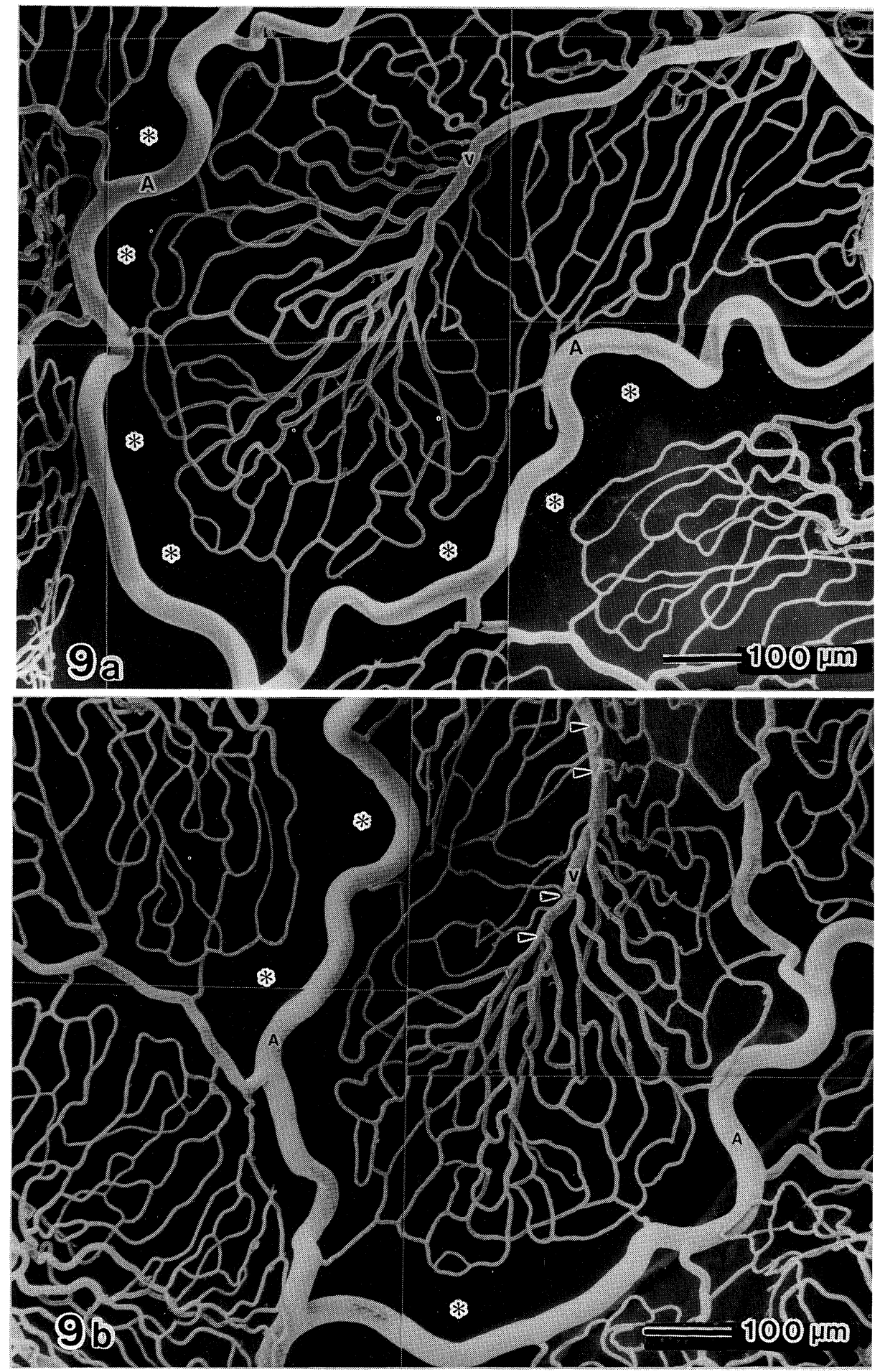


Fig. 9a and 9b. A single capillary network of the same specimen viewed from vitreous (a) and choroid (b) sides.

A single capillary network is formed by the superficial capillary network according to a disappearance of the deep network. Its meshwork is coarse, spreading in a plane with a wide CFZ (*) around arterial vessels (A), but no existence of CFZ around venous vessels $(\mathrm{V})$. Blood from the capillaries drains into venules $(\mathrm{V})$ passing in the choroid side (arrowheads).

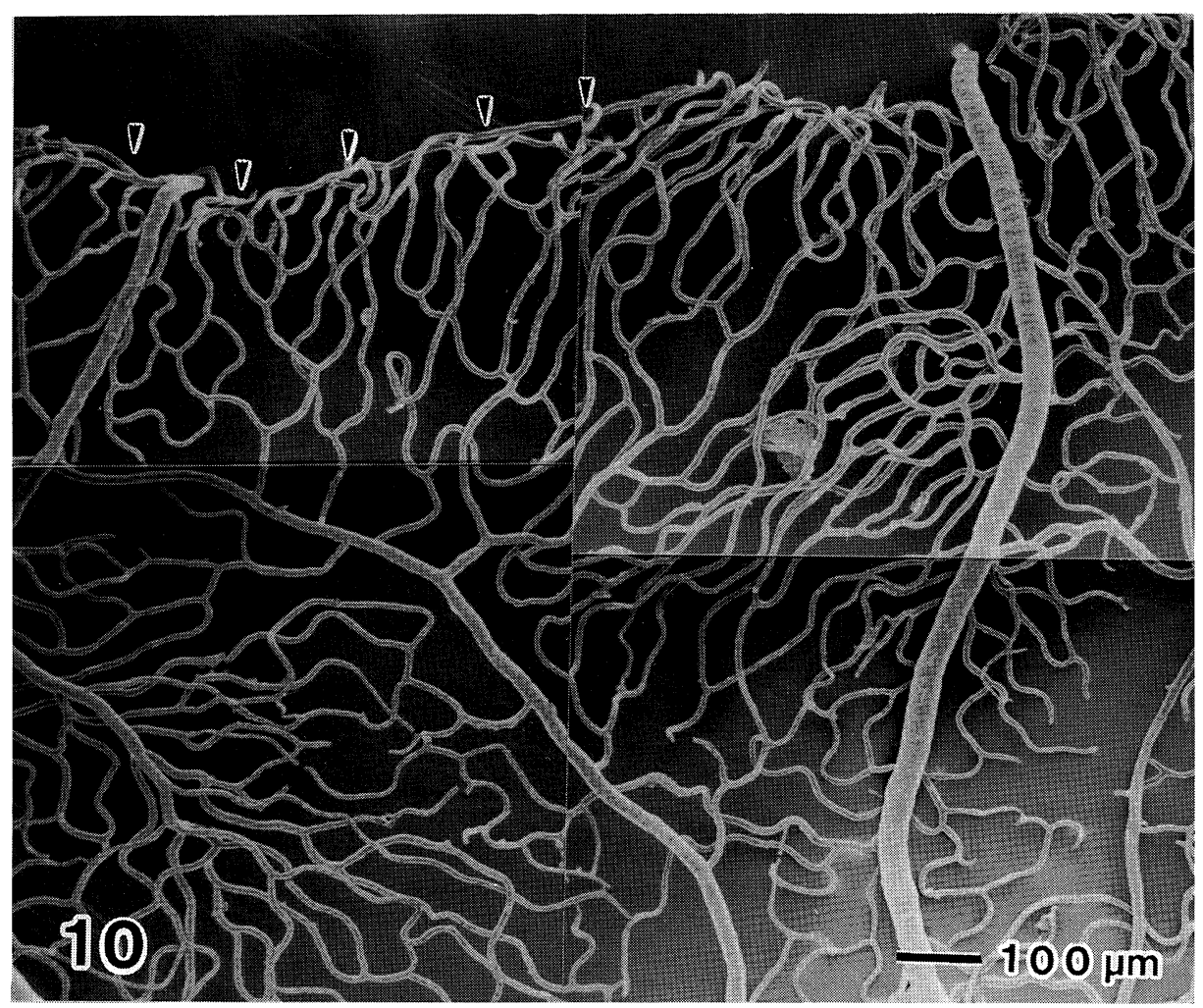

Fig. 10. SEM photograph of the serrata ora part.

Meshwork around the serrata ora is longitudinally elongated in the meridian direction, with capillary loops along the serrata ora (arrowheads).

Communication between the capillary network of the optic region of the retina and that of the optic nerve has been formed but occasionally denied in literature. Nutritious communications, however, were elucidated by demonstrating straight capillaries connecting with each other through the scleral part of the lamina cribrosa.

\section{Macula}

In this region, cell layers have disappeared in order from the superficial side, and 
the vascular architecture has also disappeared in the direction of an avascular part. According to these changes, a capillary ring surrounding the central fovea was constructed by capillaries of the deep layer without the presence of the CFZ. These capillaries appear in a concentric circular arrangement which is quite different from meshworks in other parts.

\section{Equator}

Since the CFZ was reported by His (1880), it has been understood that capillaries do not surround each arteriolar branch. However, Toussaint and Kuwabara (1961) affirmed their presence without any supporting evidence. Ujiie (1976) observed the retinal capillary architecture beneath the CFZ by microcorrosion casts. We have discussed the formation of the CFZ around the arteriole and its disappearance around the venule. Capillaries diverging from the arteriole at equal intervals always form a superficial capillary network and show an avascular boundary belt from arterioles in the same level as their network, namely, on the vitreous side. In contrast, the venule always receives blood from the deep side, namely, the choroid side. The superficial capillary network is distributed to areas around the venule. Viewed in terms of venular branches from the vitreous side, the CFZ may not appear around the venule. When the arterioles and venules were scanned from both the vitreous and choroid sides, this difference was revealed.

\section{Serrata ora}

Cell layers decrease in number and become coarse superficialward from the rod and cone lamina (choroid side), reversing the order in the macular part. Owing to this change, the vascular architecture of the deep network begins to disappear and the original double layers change to a single layer in the shape of a coarse meshwork. In the original, single-layered capillary network in the serrata ora part, the CFZ is obviously observed as a wider zone. The capillary network in the optic region of the retina does not communicate with that of the ciliary body part, and capillary loops in a regular form are directed toward the margin of the serrata ora.

\section{REFERENCES}

Anderson, D.R. (1970) Vascular supply of the optic nerve of primate. Am. J. Ophthalmol. 70, 341-351. Anderson, D.R., and Braverman, S. (1976) Re-evaluation of the optic disc vasculature, Am. J. Ophthalmol. 82, 165-174.

Araki, M. (1976) Observations on the corrosion casts of the choriocapillaries. Acta Soc. Ophthalmol. Jpn. 80, 315-326.

Ernest, J.T., and Potts, A.M. (1968) Pathophysiology of the distal portion of the optic nerve. II. Vascular relationship. Am. J. Ophthalmol. 66, 380-387. 
Henkind, P. (1967) Radial peripapillary capillaries of the retina. 1. Anatomy: Human and comparative. Brit. J. Ophthalmol. 51, 115-123.

His, W. (1880) Abbildungen über das Gefässzystem der menschlichen Netz Haut und derjeningen des Kanichens. Arch. Anat. Entwikl.

Kuwabara, T., and Cogan, D.G. (1960) Studies of retinal vascular patterns. I. Normal Architecture. Arch. Ophthal. 64, 901-911.

Lieberman, M.F., Maumenee, A.E., and Green, W.R. (1976) Histologic studies of the vasculature of the anterior optic nerve. Am. J. Ophthalmol. 82, 405-422.

Michaelson, I.C. (1954) Retinal Circulation in Man and Animals, Thomas, Springfield, Ill.

Ohta, Y., Okuda, H., Suwa, F., Okada, S., and Toda, I. (1990) Plastic injection method for preparing microvascular corrosion casts for SEM and its practical application. Okajimas Folia Anat. Jpn. 66, 301-312.

Ojima, M. (1977) Studies on the angioarchitecture of the optic nerve: 1. Relation to the ciliary arterial circulation. Acta Soc. Ophthalmol. Jpn. 81, 642-649.

Seki, R. (1987) Differences between three-dimensional posterior and peripheral retinal angioarchitecture of rats. Acta Soc. Ophthalmol. Jpn. 91, 1281-1285.

Shimizu, K. (1970) Role of radial peripapillary capillaries (RPC) in occlusive angiopathies. Photography in Ophthalmology. Int. Symp. Fluoresceine Angiography, Miami, Fla. Mod. Probl. Ophthal., 9, 7882.

Shimizu, K., and Ujiie, K. (1978) Structure of Ocular Blood Vessels, 1st Ed., Igaku-Shoin Medical Publishers, New York.

Toussaint D., and Kuwabara, T. (1961) Retinal vascular pattern. Part II. Human retinal vessels studied in three dimensions. Arch. Ophthal. 65, 575-581.

Ujiie, K. (1976) Three-dimensional view of retinal capillary. Acta Soc. Ophthalmol. Jpn. 80, 634-644.

Weiter, J.J., and Ernest, J.T. (1974) Anatomy of the choroidal vasculature. Am. J. Ophthalmol 78, 583590.

Wyber, K.C. (1956) Anastomoses between the retinal and ciliar arterial circulation. Brit. J. Ophthalmol. 40, 65-81. 